\title{
Brain metabolism changes in cases of impaired breathing or blood circulation in rodents evaluated by real time optical spectroscopy methods
}

Piavchenko, Gennadii, Seryogina, Evgeniya, Kandurova, Ksenia Yu., Shupletsov, Valery, Kozlov, Igor, et al.

Gennadii A. Piavchenko, Evgeniya S. Seryogina, Ksenia Yu. Kandurova, Valery V. Shupletsov, Igor O. Kozlov, Dmitry D. Stavtsev, Olga A. Stelmashchuk, Evgeny A. Zherebtsov, Viktor V. Dremin, Aleksander G. Alekseyev M.D., Sergey L. Kuznetsov M.D., Andrey V. Dunaev, Igor V. Meglinski, "Brain metabolism changes in cases of impaired breathing or blood circulation in rodents evaluated by real time optical spectroscopy methods," Proc. SPIE 11234, Optical Biopsy XVIII: Toward Real-Time Spectroscopic Imaging and Diagnosis, 112341E (21 February 2020); doi: $10.1117 / 12.2545708$ 


\title{
Brain metabolism changes in cases of impaired breathing or blood circulation in rodents evaluated by optical spectroscopy methods
}

\author{
Gennadii A. Piavchenko*a,b, Evgeniya S. Seryogina ${ }^{\mathrm{b}}$, Ksenia Yu. Kandurova ${ }^{\mathrm{b}}$, \\ Valery V. Shupletsov ${ }^{\mathrm{b}}$, Igor O. Kozlov ${ }^{\mathrm{b}}$, Dmitry D. Stavtsev ${ }^{\mathrm{b}}$, Olga A. Stelmashchuk ${ }^{\mathrm{b}}$, \\ Evgeny A. Zherebtsov ${ }^{\mathrm{b}, \mathrm{c}}$, Viktor V. Dremin ${ }^{\mathrm{b}, \mathrm{c}}$, Aleksander G. Alekseyev ${ }^{\mathrm{d}}$, Sergey L. Kuznetsov ${ }^{\mathrm{a}}$, \\ Andrey V. Dunaev ${ }^{\mathrm{b}, \mathrm{c}}$, and Igor V. Meglinski ${ }^{\mathrm{c}}$ \\ ${ }^{a}$ Histology, Cytology and Embryology Department, I.M. Sechenov First Moscow State Medical \\ University, Moscow, 119991, Russia \\ ${ }^{b}$ R\&D Center of Biomedical Photonics, Orel State University named after I.S. Turgenev, \\ Komsomolskaya str. 95, Orel, 302026, Russia \\ copto-Electronics and Measurement Techniques Unit, Faculty of Information and Electrical \\ Engineering, University of Oulu, 90570, Finland \\ ${ }^{\mathrm{d}}$ Anatomy, Operative Surgery and Emergency Medicine Department, Orel State University named \\ after I.S. Turgenev, Oktyabrskaya str. 25, Orel, 302028, Russia
}

\begin{abstract}
The aim of the study was to compare the metabolic activity of brain cortex after the acute hypoxia caused by the impairment of breathing or blood circulation. Male Wistar rats were randomized in two groups: impaired breathing and blood circulation failure groups. Fluorescence under 365 and $450 \mathrm{~nm}$ excitation and diffuse reflectance intensity at $550-820 \mathrm{~nm}$ range were estimated. We found that after long-term hypoxic conditions, notable metabolic changes occur. We suppose that oxygen deficiency causes an activation of the GABA shunt mechanism. In cases of blood circulation failure, fluorescence intensity changes faster than in cases of breathing impairment.
\end{abstract}

Keywords: brain metabolism, neuronal hypoxia, FAD, NADH, fluorescence spectroscopy, diffuse reflectance spectra, laser speckle contrast imaging, resuscitation procedures

\section{INTRODUCTION}

Quite often the survival of patients in the intensive care units of hospitals depends a lot on severity of disease. In modern medical diagnostics it is critically important to predict the severity of the acute statement, especially when there is a threat of brain death. As a rule, brain death is often caused by the impairment of breathing or blood circulation functions. In both of these two fundamentally different ways of dying, the organism suffers from oxygen deficiency in the cells and tissues. One of the most sensitive cells for hypoxia are the neurons of the cerebral cortex ${ }^{1}$. Mitochondria play a crucial role in processes that are central to cellular physiology, bioenergy, cell signaling, and cell death. In no type of cell are their functions as important as in neurons, where limited glycolysis makes the cell dependent on oxidative phosphorylation. Therefore, the vital activity of nerve cells is sensitive to the slightest violations of the mitochondria ${ }^{2}$.

Changes in the energy metabolism of nerve cells are evaluated by histological, histochemical and optical diagnostic methods ${ }^{3,4}$. Endoscopic fluorescence lifetime imaging (FLIM) is used to evaluate metabolic processes in the brain in patients with multiple forms of glioblastoma (GBM) in the brain in patients ${ }^{5}$. The application of the optical biopsy analysis also includes the fluorescence and diffuse reflectance spectroscopy in the ischemic brain to show a high sensitivity of brain co-enzymes involved in metabolic regulation ${ }^{6}$. Laser speckle contrast imaging allows seeing in vivo changes in the blood vessels of tissues ${ }^{7,8}$. There are studies on the application of non-contact monitoring of the cortical circulation dynamics by photoplethysmography during neurosurgical interventions ${ }^{9}$. The development of the new real-time optical biopsy techniques is relevant direction in the medical diagnostics as it provides the approach to individualize the postrehabilitation management of the patients with acute respiratory and circulatory disorders. *gennadii.piavchenko@yandex.ru; phone 7953 6144040; bmecenter.ru/en 
The aim of this study was to compare metabolic activity and structural changes in the cerebral cortex after acute hypoxia caused by impairment of respiration or blood circulation. The evaluation of metabolic activity and structural changes in the cortex was carried out by modulating an acute statement of respiratory and cardiovascular (CVS) systems impairment. Estimation of metabolic activity in cerebral cortex tissue was measured by the activity of NADH and FAD coenzymes, oxygen saturation in brain cerebral cortex - by concentration of oxy- and desoxyhemoglobin. To confirm the stop of the blood flow during respiratory and CVS disorders the LSCI methods were applied. In order to confirm the hypoxia effect on the cerebral cortex the morphological assessment of the structural post-mortal changes was made.

\section{MATERIAL AND METHODS}

For this experiment male Wistar rats aged 2 weeks (for laser speckle contrast imaging) and 2 months (for fluorescence and diffuse reflectance spectroscopy) were randomized in 2 groups: $1^{\text {st }}$ group - impaired breathing group and $2^{\text {nd }}$ group - with blood circulation failure ( $\mathrm{n}=6$ in a group). Before the experiment, animals were anaesthetized with an intramuscular injection of Zoletil ( $25 \mathrm{mg} / \mathrm{kg}$, Vibrac, France) and catheterized in right external jungular vein with PM-60 polymer catheter (SciCat, Russia, $\mathrm{d}_{\mathrm{ext}}=0.8 \mathrm{~mm}, \mathrm{~d}_{\mathrm{int}}=0.4 \mathrm{~mm}$ ). For laser speckle contrast imaging an operational access to the skull bone was carried out by dissecting of soft tissues. Operational access to the brain was carried out by drilling a window into the skull. After that, the animal head was fixed in the house-built system with stereotactic apparatus. After fixation of an anaesthetized animal the drugs that modulate a breathing or blood circulation impair were injected through the catheter. For breathing failure model, a combination of Propofol $0.3 \mathrm{ml}$ with lethal dose of cisatracurium injected immediately causing a spasm of the respiratory muscles and respiratory arrest. For brain blood circulation failure model lidocaine solution (2\%) in a dose of $1 \mathrm{ml}$ was selected because of its properties to immediately cause a cardiac arrest.

The setup for laser speckle contrast imaging consisted of a compact laser module LDM785 with a working wavelength of $785 \mathrm{~nm}$ and a power of $20 \mathrm{~mW}$ (Thorlabs, USA), a diffuser DG10-1500 (Thorlabs, USA), a polarizer LPNIRE100-B (Thorlabs, USA) and a holder for a polarizer RSP1/M (Thorlabs, USA), as well as a fixed focus lens MVL25TM23 (Thorlabs, USA). Blood flow dynamics was recorded with IDS-3260CP-M-GL R2 camera (IDS Imaging Development Systems GmbH, Germany) ${ }^{10}$.

Fluorescence spectra were obtained under 365 and $450 \mathrm{~nm}$ excitation wavelengths. The diffuse reflectance spectra channel included HL-2000-FHSA Tungsten Halogen Light Source ("Ocean Optics", USA) with a wavelength range of 360-2400 $\mathrm{nm}$. The light collected from the tissue was filtered by $400 \mathrm{~nm}$ and $495 \mathrm{~nm}$ optical filters to attenuate backscattered radiation and then it was analyzed by a CCD Flame Spectrometer ("Ocean Optics", USA) in the range of 350-1000 $\mathrm{nm}^{11}$. To avoid photobleaching effect, fluorescence spectra were recorded 1-2 time per minute with the excitation laser turned off between the measurements. System control and further data processing were performed using a personal computer with customdeveloped software in Matlab ${ }^{\circledR}$ program environment.

After measurements, the brain was fixed in buffered formalin for histological analysis. The $5 \mu \mathrm{m}$ thick slides stained with hematoxylin and eosin were analyzed for cortical hypoxia signs. The rat motor cortex as a region that takes part in the regulation of motor behavior ${ }^{12}$ was identified by Paxinos and Watson Rat Brain Stereotaxic Atlas ${ }^{13}$. The study was conducted according to the "Principles of Good Laboratory Practice"14. Manipulations with animals, as well as the conditions, were reviewed and approved by the Ethical Commission of Orel State University named after I.S. Turgenev. Rats were kept in controlled environmental conditions $\left(20-26^{\circ} \mathrm{C}\right.$ at a relative humidity of $\left.30-70 \%\right)$. The temperature and humidity in the rooms were monitored daily using automatic electronic thermometers and psychrometers. A 12-hour lighting cycle and a 10-times exchange of the room air volume per hour was maintained in the rooms.

\section{EXPERIMENTAL RESULTS AND DISCUSSION}

Figure 2 features the images recorded with laser speckle contrast imaging, as well as the analysis of speckle contrast dynamics during the experiments. Laser speckle contrast analysis shows that blood flow process stops in the cortical tissue during the first minute in case of impaired blood circulation and 2 minutes for impaired respiration. The obtained images were processed by a custom-developed algorithm in the offline regime using a Matlab ${ }^{\odot}$ software environment. The temporal algorithm has been used for speckle contrast images obtaining. Totally, 40 speckle contrast images have been 
calculated and averaged in every speckle contrast image. Thus, the calculation of the final speckle contrast image was carried out according to the Eq. 1:

$$
K=\frac{\sigma_{N}}{\langle I\rangle_{N}}
$$

where \langle\rangle - the symbol of averaging; $N$ - the window of averaging $N \times N(N=40) ;\langle I\rangle_{N}$-average intensity in the window $N \mathrm{x} N ; \sigma_{N}-$ standard deviation in the window $N \mathrm{x} N$.
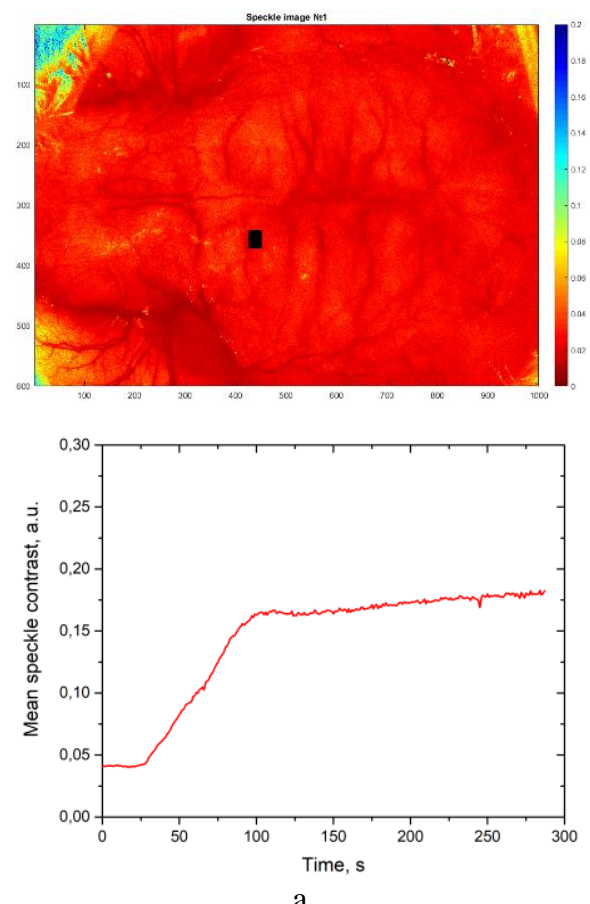

a
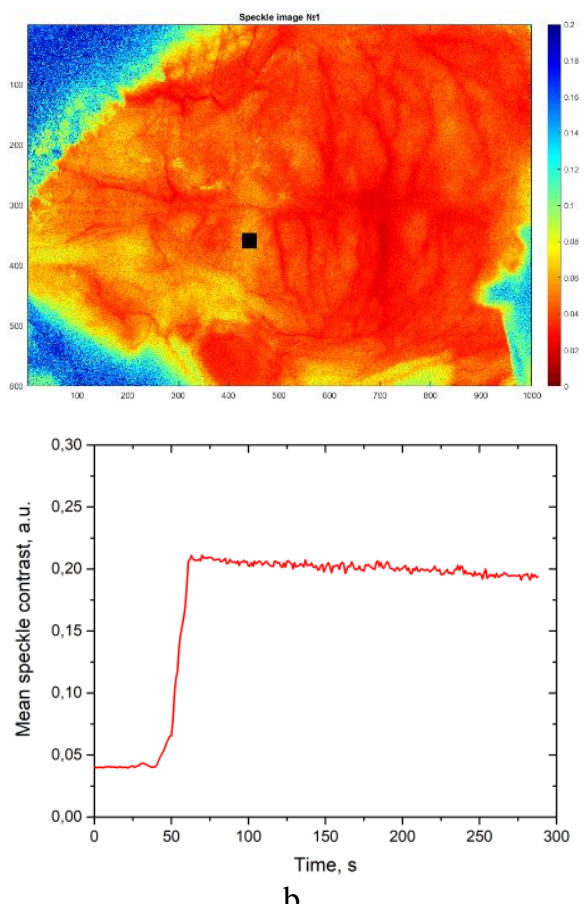

b

Figure 2. Laser speckle contrast images and mean speckle contrast analysis in cases of impaired respiration (a) and blood circulation (b) in the microcirculatory vessels. Black shapes represent regions of interest for speckle contrast averaging.

To analyze the dynamics of the fluorescence, the maximum intensities of obtained fluorescence spectra $I F_{N}$ were normalized by the base value recorded before the administration of the solutions $I F_{0}$ (Fig. 3a and 4a). The intensities of diffuse reflectance at the wavelengths corresponding with the light absorption by deoxyhemoglobin $(560 \mathrm{~nm})$ and oxyhemoglobin $(580 \mathrm{~nm}$ ) were analyzed by the same algorithm (Fig. 3b and $4 \mathrm{~b}$ ) and used for additional normalization of fluorescence spectroscopy data to compensate the absorbing effect of blood on recorded data. 


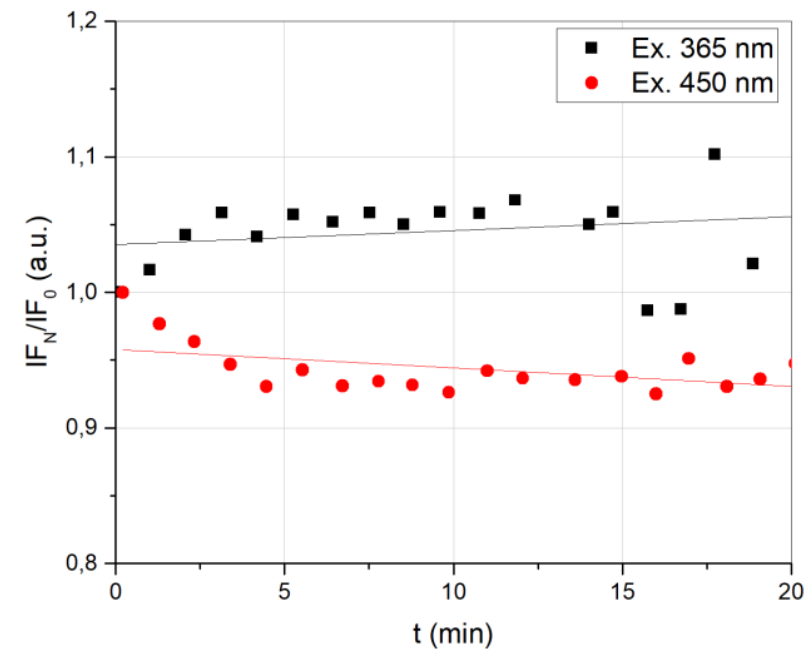

a)

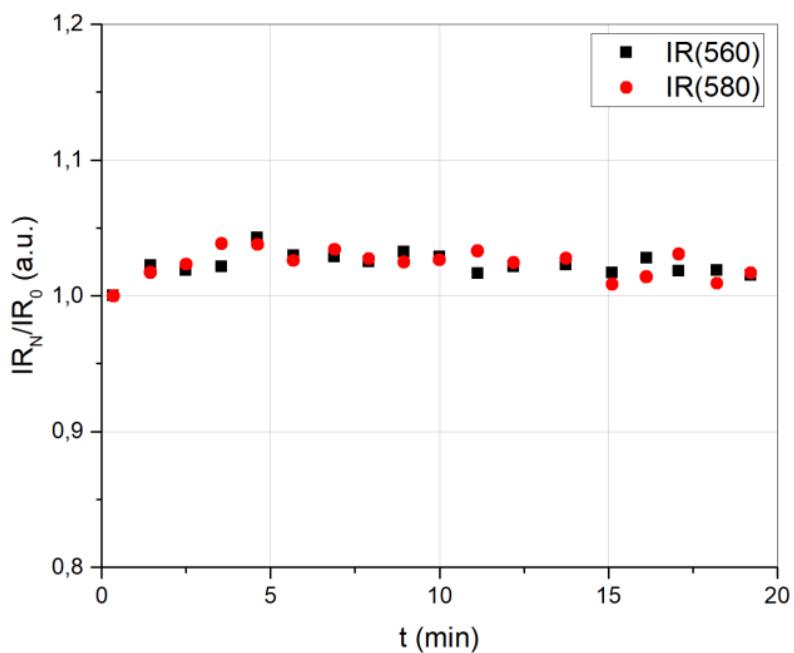

b)

Figure 3. Respiration failure model. Typical fluorescence intensity dynamics under $365 \mathrm{~nm}$ and $450 \mathrm{~nm}$ excitation (a). Typical diffuse reflectance dynamics (b).

Fluorescence spectra analysis showed that in both groups, the fluorescence intensity corresponding to FAD coenzyme decreased. At the same time, the increase of $365 \mathrm{~nm}$-induced fluorescence associated with NADH was observed.

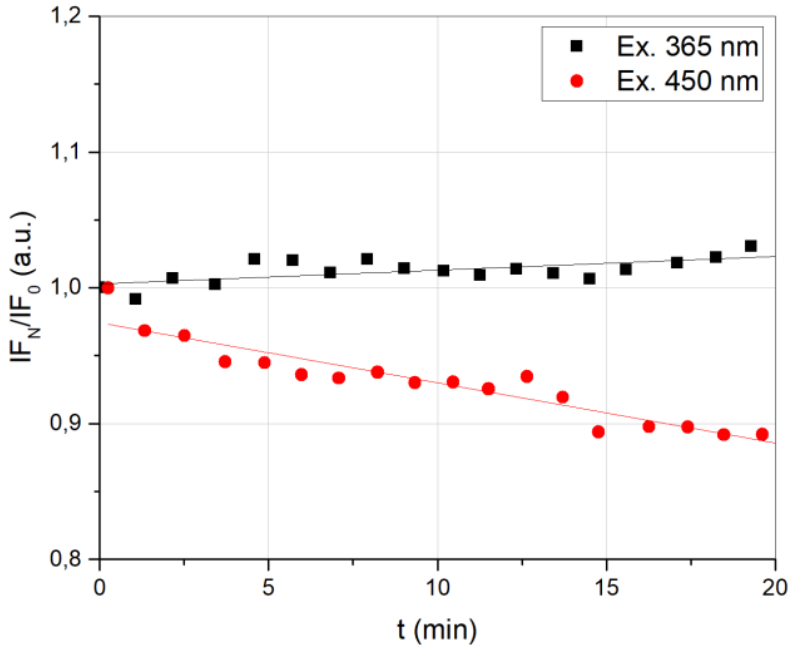

a)

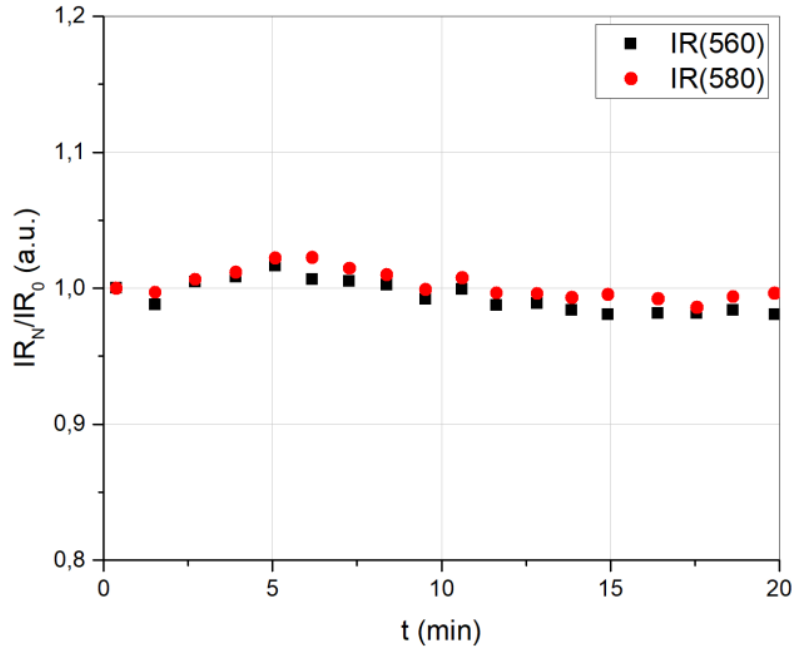

b)

Figure 4. Blood circulation impairment model. Typical fluorescence intensity under $365 \mathrm{~nm}$ and $450 \mathrm{~nm}$ excitation (a). Typical diffuse reflectance intensity dynamics (b).

The linear fitting was applied to assess the trend and the rate of fluorescence intensity dynamics. It was found that in case of blood circulation failure the tissue ischemia occurs more acute, which is demonstrated by the growth of NADH and decrease of FAD activity (Fig. 3a and 4a). The analysis of specific diffuse reflectance intensities corresponding with the presence of oxygenated and deoxygenated blood showed that mostly the presence of deoxyhemoglobin was higher than oxyhemoglobin. Diffuse reflectance intensities demonstrate slight changes as compared to the fluorescence ones (Fig. 3b and $4 \mathrm{~b}$ ), which indicates that the amount of blood was almost the same during the experiment while the metabolic changes in tissues occurred.

In the histological slides, we found that in all the animals of the first group, the signs of focal perivascular edema, slight hypoxic changes in the morphology of neurons (Fig. 5a). In the second group in all tissue sections, we found a significant 
number of hyperchromic wrinkled neurons, glial nodules at the site of necrotic neurons, as well as pericellular edema (Fig. $5 b)$.

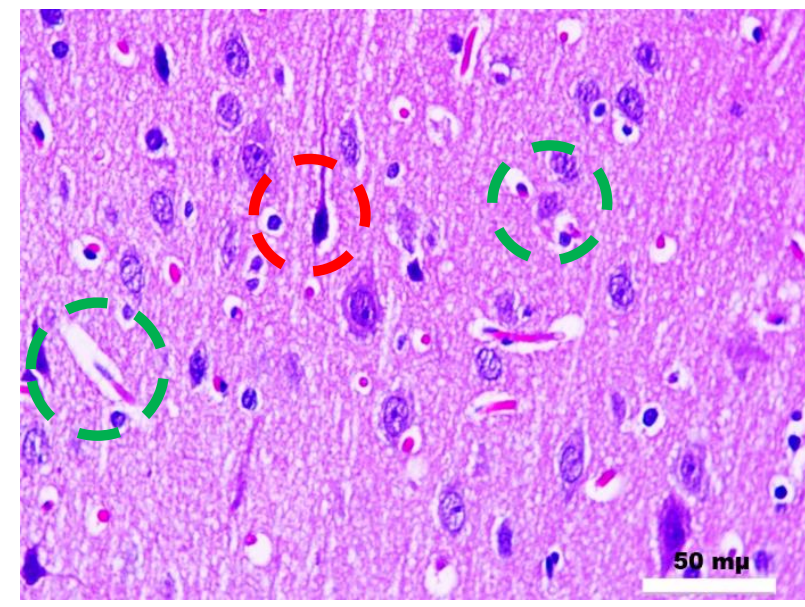

a)

Motor cortex sections. H\&E stain. Signs of numeric hypoxic changes of the neuronal morphology (red) and local perivascular edema (green)

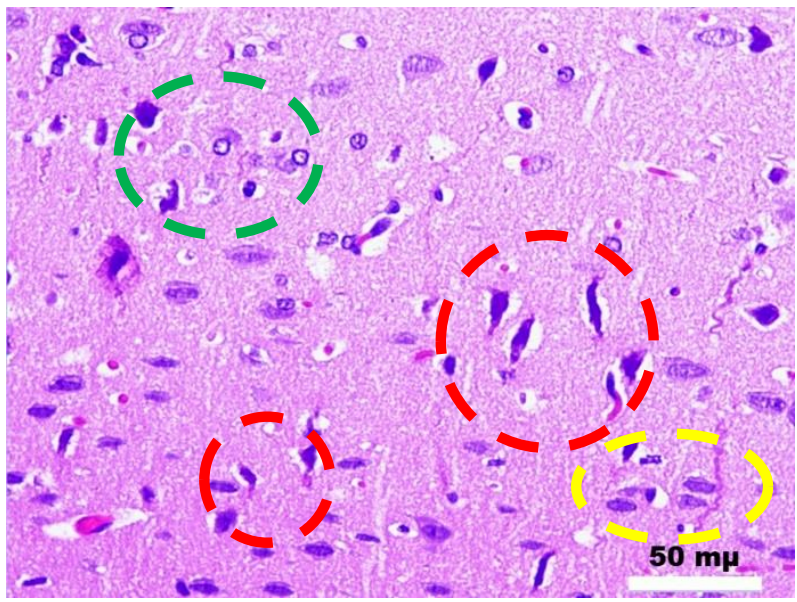

b)

Motor cortex sections. H\&E stain. Huge amount of hyperchromic wrinkled neurons (red), pericellular edema (green), glial nodules \& necrobiotic neurons (yellow)

Figure 5. Rat brain motor cortex sections for the $1^{\text {st }}$ (a) and $2^{\text {nd }}$ groups (b). H\&E stain.

The results show that strong oxygen deficiency causes an increase in NADH and decrease of FAD intensity. This may be explained by the activation of the GABA shunt mechanism (succinate formation from GABA) ${ }^{15,16}$. It happens because of cerebral cortex contains a lot of GABAergic neurons which being under substantial hypoxia shift the biochemical processes to the energy economy statement. The increase of deoxyhemoglobin shows the shift of the process from the Krebs cycle to the GABA shunt mechanism ${ }^{17}$.

In cases of blood circulation failure, we found that fluorescence intensity changes faster than in cases of breathing impairment. We conclude that acute cardiovascular failure (e.g. sudden cardiac arrest) is a more life-threatening process that causes a faster increase of neuronal metabolism deficiency in comparison with acute respiratory system disorders. After long time hypoxia, the biochemical processes shift to the low energy regime and help an organism to resist hypoxia until the state of full anoxia.

In the experiment, we found that long-term brain hypoxia causes the changes in coferment functioning as well as regulates GABA associated cortical activity ${ }^{18}$.

In acute respiratory failure, there is a gradual increase in hypoxic disorders and a slowdown of hemodynamic processes. In acute circulatory disorders, there is a sharp cessation of the oxygenated blood supply to the brain cortex structures.

\section{CONCLUSION}

The blood circulation in the vessels of the cerebral cortex stops in case of respiratory arrest after $\sim 2$ minutes, and when heart arrest occurs - after $\sim 1$ minute. Changes in the brain tissue oxygen saturation under a heart arrest happen in the first 5 minutes, while acute breathing failure practically does not change it. Heart failure is characterized by more acute hypoxic disorders in the cerebral cortex in comparison with processes that occur in the cortex when breathing is impaired. Acute cardiovascular failure is a more life-threatening process that causes a rapid increase in the neuronal metabolic deficit in compare with acute respiratory diseases. This may be used to personalize the management of patients after acute circulatory disorders and respiratory arrest in the prognosis and rehabilitation.

\section{ACKNOWLEDGMENTS}

This study was supported by the Russian Science Foundation under project No.18-15-00201. E.Z. acknowledges funding from the grant of the Academy of Finland (grant No. 318281), A.D. and I.M. acknowledge funding from the Academy of Finland (grant No. 326204). G.P. acknowledges Dr. Konstantin N. Lapin for catheterization guiding and useful discussion. 


\section{REFERENCES}

[1] Stelmashchuk, O., Seryogina, E., Piavchenko, G., Alekseyev, A., Zherebtsov, E., Dunaev, A., Rafailov, I., Vorobyev, E., Kuznetsova, A., Kuznetsova, E. and Rafailov, E., "Use of fluorescent optical fibre probe for recording parameters of brain metabolism in rat model," Proc.SPIE 10685, 106854R (2018).

[2] Gandhi, S. and Abramov, A. Y., "Mechanism of oxidative stress in neurodegeneration," Oxid. Med. Cell. Longev. 2012, 428010 (2012).

[3] Gulyaeva, N. V., Stepanichev, M. Y., Onufriev, M. V., Lazareva, N. A., Zarzhetsky, Y. V., Gurvitch, A. M. and Volkov, A. V., "Cardiac arrest induces decrease of nitric oxide synthase activity and increase of free radical generation in rat brain regions," Neurosci. Lett. 220(3), 147-150 (1996).

[4] Angelova, P. R., Kasymov, V., Christie, I., Sheikhbahaei, S., Turovsky, E., Marina, N., Korsak, A., Zwicker, J., Teschemacher, A. G., Ackland, G. L., Funk, G. D., Kasparov, S., Abramov, A. Y. and Gourine, A. V., "Functional oxygen sensitivity of astrocytes," J. Neurosci. 35(29), 10460-10473 (2015).

[5] Hatami, N., "Fluorescence lifetime imaging microscopy for brain tumor image-guided surgery," J. Biomed. Optis 15(5), 056022 (2010).

[6] Dunaev, A. V, Dremin, V. V, Zherebtsov, E. A., Rafailov, I. E., Litvinova, K. S., Palmer, S. G., Stewart, N. A., Sokolovski, S. G. and Rafailov, E. U., "Individual variability analysis of fluorescence parameters measured in skin with different levels of nutritive blood flow," Med. Eng. Phys. 37(6), 574-583 (2015).

[7] Kalchenko, V., Sdobnov, A., Meglinski, I., Kuznetsov, Y., Molodij, G. and Harmelin, A., “A robust method for adjustment of laser speckle contrast imaging during transcranial mouse brain visualization," Photonics 6(80), 1-9 (2019).

[8] He, J., Lu, H., Deng, R., Young, L., Tong, S. and Jia, X., "Real-time monitoring of cerebral blood flow by laser speckle contrast imaging after cardiac arrest in rat," Proc. Annu. Int. Conf. IEEE Eng. Med. Biol. Soc. EMBS 2015, 6971-6974 (2015).

[9] Lyubashina, O. A., Mamontov, O. V, Volynsky, M. A., Zaytsev, V. V and Kamshilin, A. A., "Contactless Assessment of Cerebral Autoregulation by Photoplethysmographic Imaging at Green Illumination," Front. Neurosci. 13, 1235 (2019).

[10] Potapova, E. V, Seregina, E. S., Dremin, V. V, Stavtsev, D. D., Kozlov, I. O., Zherebtsov, E. A., Mamoshin, A. V, Ivanov, Y. V and Dunaev, A. V., "Laser speckle-contrast imaging of blood microcirculation in pancreatic tissues during laparoscopic interventions," Quantum Electron. 50(1), 33-40 (2020).

[11] Dremin, V. V., Potapova, E., Zherebtsov, E., Kozlov, I., Seryogina, E., Kandurova, K., Alekseyev, A., Piavchenko, G., Kuznetsov, S., Mamoshin, A. and Dunaev, A., "Optical fine-needle aspiration biopsy in a rat model," Proc. SPIE 10877, 108770K (2019).

[12] P'yavchenko, G. A., Shmarkova, L. I. and Nozdrin, V. I., "Changes in the Number of Neurons in the Rat Motor Cortex and Movement Activity with Age,” Neurosci. Behav. Physiol. 46(3), 270-273 (2016).

[13] Paxinos, G. and Watson, C., [The Rat Brain in Stereotaxic Coordinates Sixth Edition] (2007).

[14] Environment Directorate OECD., "OECD series on principles of good laboratory practice and compliance monitoring,” Ann. Ist. Super. Sanita 33(1), 1-172 (1997).

[15] Hassel, B., Johannessen, C. U., Sonnewald, U. and Fonnum, F., "Quantification of the GABA Shunt and the Importance of the GABA Shunt Versus the 2-Oxoglutarate Dehydrogenase Pathway in GABAergic Neurons," J. Neurochem. 71(4), 1511-1518 (2002).

[16] Wagner, K. R., Kleinholz, M. and Myers, R. E., "Delayed Neurologic Deterioration Following Anoxia: Brain Mitochondrial and Metabolic Correlates," J. Neurochem. 55(5), 1407-1417 (1989).

[17] Gerich, F. J., Hepp, S., Probst, I. and Müller, M., "Mitochondrial inhibition prior to oxygen-withdrawal 
facilitates the occurrence of hypoxia-induced spreading depression in rat hippocampal slices," J. Neurophysiol. 96(1), 492-504 (2006).

[18] Clarkson, A. N., Clarkson, J., Jackson, D. M. and Sammut, I. A., "Mitochondrial involvement in transhemispheric diaschisis following hypoxia-ischemia: Clomethiazole-mediated amelioration," Neuroscience 144(2), 547-561 (2007). 